\title{
Multiplexed, high-throughput measurements of cell contraction and endothelial barrier function
}

\author{
Ramin Rokhzan ${ }^{1,2} \cdot$ Chandra C. Ghosh $^{1,3,4} \cdot$ Niccole Schaible $^{1,3} \cdot$ Jacob Notbohm $^{5} \cdot$ Haruka Yoshie $^{6}$. \\ Allen J. Ehrlicher ${ }^{6} \cdot$ Sarah J. Higgins $^{1} \cdot$ Ruyuan Zhang ${ }^{1,7} \cdot$ Hermann Haller $^{2} \cdot$ Corey C. Hardin ${ }^{8} \cdot$ Sascha David $^{2}$. \\ Samir M. Parikh $\mathbb{1}^{1,9} \cdot$ Ramaswamy Krishnan ${ }^{1,3}$
}

Received: 17 April 2018 / Revised: 20 August 2018 / Accepted: 31 August 2018 / Published online: 11 October 2018

(c) United States \& Canadian Academy of Pathology 2018

\begin{abstract}
Vascular leakage, protein exudation, and edema formation are events commonly triggered by inflammation and facilitated by gaps that form between adjacent endothelial cells (ECs) of the vasculature. In such paracellular gap formation, the role of EC contraction is widely implicated, and even therapeutically targeted. However, related measurement approaches remain slow, tedious, and complex to perform. Here, we have developed a multiplexed, high-throughput screen to simultaneously quantify paracellular gaps, EC contractile forces, and to visualize F-actin stress fibers, and VE-cadherin. As proof-of-principle, we examined barrier-protective mechanisms of the Rho-associated kinase inhibitor, Y-27632, and the canonical agonist of the Tie2 receptor, Angiopoietin-1 (Angpt-1). Y-27632 reduced EC contraction and actin stress fiber formation, whereas Angpt-1 did not. Yet both agents reduced thrombin-, LPS-, and TNF $\alpha$-induced paracellular gap formation. This unexpected result suggests that Angpt-1 can achieve barrier defense without reducing EC contraction, a mechanism that has not been previously described. This insight was enabled by the multiplex nature of the force-based platform. The high-throughput format we describe should accelerate both mechanistic studies and the screening of pharmacological modulators of endothelial barrier function.
\end{abstract}

These authors contributed equally: Samir M. Parikh, Ramaswamy Krishnan.

Electronic supplementary material The online version of this article (https://doi.org/10.1038/s41374-018-0136-2) contains supplementary material, which is available to authorized users.

Ramaswamy Krishnan

rkrishn2@bidmc.harvard.edu

1 Center for Vascular Biology Research, Beth Israel Deaconess Medical Center and Harvard Medical School, Boston, MA, USA

2 Division of Nephrology and Hypertension, Hannover Medical School, Hannover, Germany

3 Department of Emergency Medicine, Beth Israel Deaconess Medical Center and Harvard Medical School, Boston, MA, USA

4 Transplant Research Program, Division of Nephrology, Department of Medicine, Boston Children's Hospital and Harvard Medical School, Boston, MA, USA

\section{Introduction}

Across several major diseases including acute lung injury, atherosclerosis, tumor neovascularization, hypertension, diabetes mellitus, and coronary artery disease, the endothelial barrier becomes disrupted through the formation of paracellular gaps [1-8]. Since the pioneering work of Guido Majno, and George Palade in the 1960s [9, 10], paracellular gaps in response to inflammatory mediators have been considered to arise by contractile forces that act to pull

5 Department of Engineering Physics, University of Wisconsin, Madison, WI, USA

6 Department of Bioengineering, McGill University, Montreal, QC, Canada

7 Department of Critical Care Medicine, Rui Jin Hospital, Shanghai Jiao Tong University School of Medicine, Shanghai, China

8 Department of Pulmonary and Critical Care, Massachusetts General Hospital, Boston, USA

9 Division of Nephrology and Department of Medicine, Beth Israel Deaconess Medical Center and Harvard Medical School, Boston, MA, USA 
intercellular junctions apart [11-22]. These forces are generated via acto-myosin interactions and manifest upon the EC substrate as tractions [12, 18-21, 23, 24].

Endothelial barrier function is routinely measured through changes in electrical resistance or macromolecular permeability across a confluent monolayer [25]. While these measurements are fast, they cannot simultaneously quantify underlying biophysical forces. As a result, further validation requires follow-up measurements of EC contraction using single cells [21], cell doublets [20,23], micropatterned cell clusters [12, 18], and continuous cell monolayers [19, 24]. These follow-up measurements are slow, complex, and lowthroughput, limiting the pace of mechanistic insights and the breadth of pharmacological investigation.

Here, we have developed "mini-XPerT", a force-based high-throughput assay that combines monolayer traction microscopy $[19,26]$ to measure EC contraction, Express Permeability Testing (XPerT) [27] to measure paracellular gaps, and immunofluorescence imaging to measure structural and morphological changes. These measurements are miniaturized in 96-well plates using the approach of contractile force screening [28, 29]. Mini-XPerT utilizes elastic silicone substrates that are tunable in Young's modulus $(0.3-150 \mathrm{kPa})$ [29], thereby enabling studies spanning the entire known (patho)physiological range of substrate stiffness. Additional practical advantages of mini-XPerT include the following: it is non-invasive, non-destructive, and suitable for both short- and long-term assessment of barrier function.

Applying mini-XPerT, we have studied monolayers of primary human dermal microvascular ECs subjected to acute and progressive mediators of pathological permeability. We have compared barrier-protective mechanisms of two well-known and mechanistically distinct barrierprotective agents, the Rho-kinase inhibitor Y-26732 [30] and the canonical Tie2 receptor agonist Angiopoietin-1 (Angpt-1) [31]. The multiplexed nature of mini-XPerT unveiled evidence to suggest that Angpt-1 achieves barrier protection without diminishing contractile forces. Taken together, these results reveal that the endothelium can protect itself by: (1) reorganizing the actin cytoskeleton-a contraction-dependent mechanism-as in the case of Y27632 or (2) by accumulating VE-cadherin to fortify cell-cell junctions-a contraction-independent mechanism -as in the case of Angpt-1.

\section{Material and methods}

While our methodologies are generalizable across adherent cell types for a wide spectrum of experimental conditions, we focused here on a representative endothelial cell type, a commonly studied range of substrate stiffness values, and a commonly used extracellular matrix ligand, collagen I.

\section{Cell culture}

All experiments were performed using primary dermal human microvascular endothelial cells (Lonza, Basel, Switzerland) at passages 4-7 in EGM2-MV medium (Lonza, Basel, Switzerland) containing $4.7 \%$ fetal bovine serum. The cells were plated for 2-3 days prior to experimentation.

\section{Fabrication of multi-well plates}

Soft, elastic (Young's Modulus $=0.3$ or $3 \mathrm{kPa}$ ), and deformable substrates were prepared using $\mathrm{NuSil}^{\circledR} 8100$ (NuSil Silicone Technologies, Carpinteria, CA) in 96-well plates [29]. Embedded in the substrate surface are fluorescent beads (diameter $=\sim 400 \mathrm{~nm}$ ) whose displacements enable traction force calculations. Ligated to the substrate surface is biotinylated collagen I (collagen I, Advanced Biomatrix, Carlsbad, CA; 10x PBS, Corning Life Sciences, Tewksbury, MA; EZ-Link NHS-LC-LC-Biotin, Thermo Fisher Scientific, Waltham, MA) whose binding with FITCavidin enables the identification of paracellular gaps. Detailed information of plate preparation, ligation, and cell seeding is provided in the supplemental material.

\section{Measurement of cellular contractile forces: monolayer traction microscopy}

The local contraction that an endothelial cell exerts upon its substrate is called traction [26, 32]. To measure tractions, we utilized an inverted epi-fluorescence microscope (DMI 6000B, Leica Inc., Germany) equipped with a heated chamber $\left(37^{\circ} \mathrm{C}\right)$, a monochrome camera (Leica DFC365 FX), and a motorized stage. We recorded spatial images of substrateembedded fluorescent beads at 10x magnification. Based on the bead displacements (resolution $=\sim 15 \mu \mathrm{m}$ ) relative to a cell-free image, together with knowledge of substrate stiffness and thickness, we computed tractions using the approach of Fourier Transform Traction Cytometry [33], modified to the case of cell monolayers [26]. From each traction map, we calculated the root-mean squared traction (RMST) value and report this as a measure of average contraction in the monolayer. On a well-by-well basis, we first computed the ratio of the RMST after vs. before stimulation. We then normalized all values of a given treatment group to the mean of the corresponding vehicle control group and further calculated the average of each treatment group.

This permits us to compare differences across treatment conditions in each 96-well plate as well as across plates. 


\section{Measurement of paracellular gaps: Express Permeability Testing (XPerT)}

At the end of drug treatment, FITC-conjugated avidin dissolved in PBS (Thermo Fisher Scientific, Waltham, MA) was added to each well and incubated for $210 \mathrm{~s}$. The cellular monolayer acts as a mask; Avidin binds to biotinylated collagen I only in areas of paracellular gaps [27]. When further visualized using an inverted epi-fluorescence microscope (DMI 6000B, Leica Inc., Germany, 10x magnification), the FITC label localizes the gap and thereby permits quantitation of both its location and overall incidence in a well. The raw FITC images were converted into 8-bit binary images; the same threshold value was applied to all images in the manuscript. Gaps were computed from the areas in white and expressed as the percentage of the total area in a given image. We then normalized all values of a given treatment group to the mean of the corresponding vehicle control group and further calculated the average of each treatment group. To perform these analyses in highthroughput, a custom-macro was written using Fiji software. The macro is provided in the supplemental material.

\section{Quantification of F-actin stress fiber expression and orientation}

At the end of drug treatment, the cells were fixed with $3 \%$ Formalin (Sigma-Aldrich, St. Louis, MO) for $5 \mathrm{~min}$ and then washed three times with PBS. Alexa Fluor 480 phalloidin (Thermo Fisher Scientific, Waltham, MA) at a concentration of 1:300 and Hoechst dye (Thermo Fisher Scientific, Waltham, MA) at a concentration of 1:2000 was added together to each well for $30 \mathrm{~min}$. The wells were washed twice with PBS and visualized using an inverted epi-fluorescence microscope (DMI 6000B, Leica Inc., Germany, 20x magnification). The Alexa 480 labels the actin stress fibers while the Hoechst dye labels the nuclei. From each image, we chose at random, six different regions. In these regions, we quantified F-actin orientation (in terms of anisotropy) using an open source plugin called Fibriltool [34] for Fiji. We also quantified corresponding F-actin expression (in terms of mean-fluorescent intensity) in those same regions. Across all images for a given treatment group, we normalized all values to the mean of the corresponding vehicle control group and further calculated the average of each treatment group.

\section{Evaluation of VE-cadherin expression}

At the end of drug treatment, the cells were fixed with $3 \%$ Formalin for $5 \mathrm{~min}$, washed three times with PBS, and then permeabilized with $0.1 \%$ saponin (Sigma-
Aldrich, St. Louis, MO) for 5 min. The cells were washed with PBS and a mouse-derived monoclonal antiVE-cadherin antibody (BD Biosciences, Franklin Lakes, $\mathrm{NJ}$ ) at a concentration of 1:250 was added to each well for $2 \mathrm{~h}$. The wells were washed twice with PBS and a secondary antibody conjugated with FITC (Thermo Fisher Scientific, Waltham, MA) at a concentration of 1:500 and Hoechst dye (Thermo Fisher Scientific, Waltham, MA) at a concentration of 1:2000 was added together for an additional $30 \mathrm{~min}$. The cells were then washed twice with PBS. When visualized using an inverted epi-fluorescence microscope (DMI 6000B, Leica Inc., Germany, 20x magnification), the FITCconjugated antibody labels VE-cadherin expression at the cell-cell junction and the Hoechst dye labels nuclei.

\section{Measurement of trans-endothelial electrical resistance (TEER)}

An electric cell-substrate impedance sensing system (ECIS, Applied BioPhysics Inc., Troy, NY) was used to measure trans-endothelial electrical resistance of EC monolayers [35]. Briefly, cells were seeded on collagen I coated electrode arrays $(8 \mathrm{~W} 10 \mathrm{E}+$, Applied BioPhysics Inc., Troy, $\mathrm{NY}$ ) and grown to confluency $(24-48 \mathrm{~h})$, determined by achieving electric resistance of $>1500 \mathrm{ohm}$. Cells were then incubated with indicated drugs or EBM-2 medium alone as a vehicle control with raw resistance continually measured over time at an amplitude of $4000 \mathrm{~Hz}$. For every well, raw resistance values were divided by the correspondent timezero raw resistance value to obtain the normalized TEER.

\section{Drug treatments}

EBM-2 basal medium served as vehicle for drug treatments, and as the vehicle control. Angiopoietin-1, CD14 and LPS binding protein were purchased from R\&D Systems (Minneapolis, MN). Thrombin was purchased from EMD Millipore (Billerica, MA). LPS serotype O111:B4 was purchased from Sigma-Aldrich (St. Louis, MO). Y-27632 was purchased from EMD Biosciences (LaJolla, CA).

\section{Statistics}

Data are presented as mean \pm SEM. For gaps, ECIS measurements, F-actin intensity and anisotropy measurements, statistical significance was tested by two-tailed unpaired $t$-test with Welch's correction. For traction measurements, the more appropriate non-parametric two-tailed Mann-Whitney $U$-test for data deviating from Gaussian distribution was used. A $p$-value $<0.05$ was considered significant. 


\section{Results and discussion}

A key downstream effector of EC barrier disruption is the Rho-associated protein kinase, ROCK [36-38]. Activation of ROCK signaling promotes actin stress fiber formation, myosin light chain phosphorylation, endothelial cell contraction and, ultimately, paracellular gap formation [15, 30, 36-42]. Conversely, inhibition of ROCK signaling via the pharmacological agent Y-27632 promotes endothelial barrier defense $[12,30]$.

We chose Y-27632 as an exemplar to evaluate miniXPerT (Fig. 1). Based on typically used concentrations

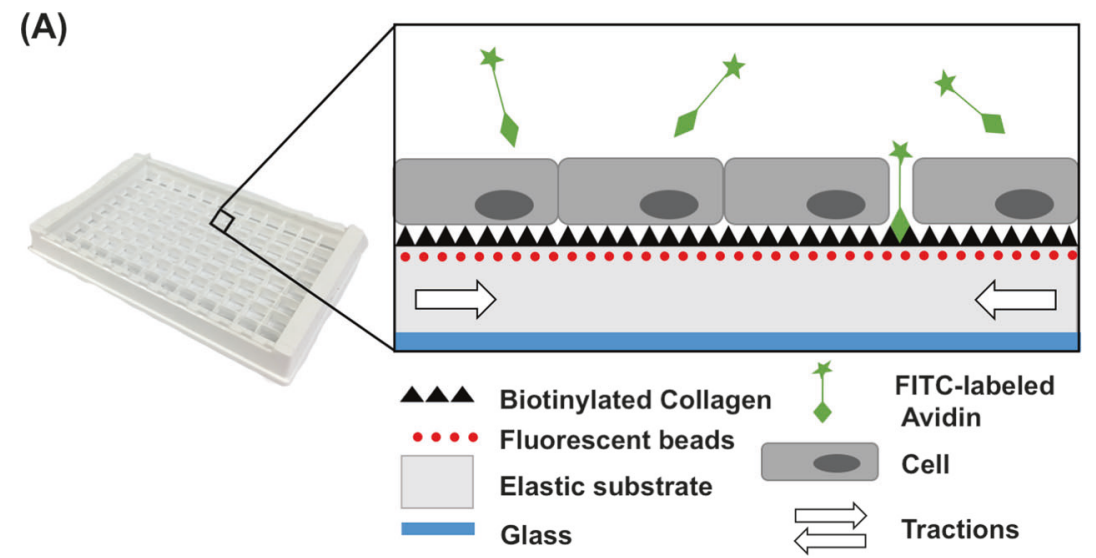

(B)
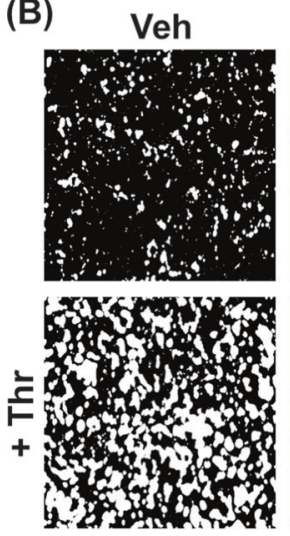

Y-27632
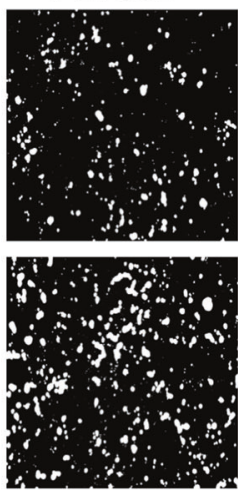

(D)

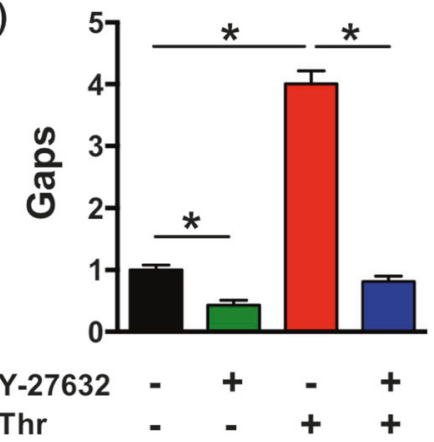

Fig. 1 Mini-XPerT-simultaneous measurements of barrier function and cellular contractile forces in a high-throughput format. A Siliconebased elastic substrates (Young's Modulus $=0.3 \mathrm{kPa}$ or $3 \mathrm{kPa}$ ) were prepared in a 96-well plate format. Embedded in the substrate are fluorescent beads (diameter $=\sim 400 \mathrm{~nm}$; shown in red) whose displacement enables the computation of monolayer contractile forces (shown as white arrows). Ligated to the substrate is biotinylated collagen I (shown as black triangles) whose binding with FITC-avidin (shown in green) enables the identification of paracellular gaps. B Representative gap images for every experimental condition,
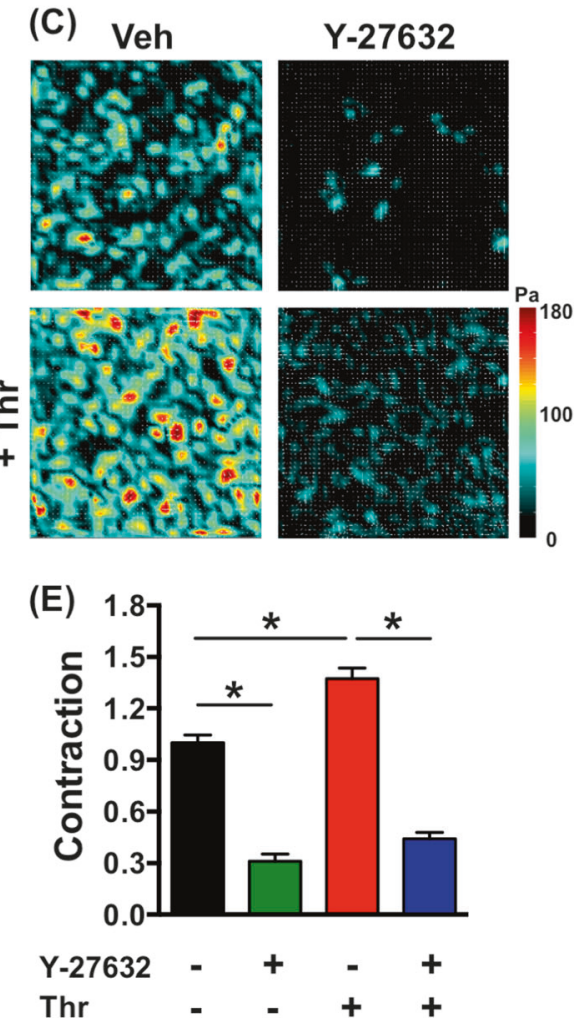

C corresponding traction force maps, and D-E averaged values. While gap formation and contraction were increased with thrombin $(1 \mathrm{U} / \mathrm{ml}$, $30 \mathrm{~min}$ ) it was significantly reduced by pre-treatment for $20 \mathrm{~min}$ with the ROCK inhibitor, Y-27632 $(5 \mu \mathrm{M})$. In $\mathbf{D}$, plotted is the gap area normalized to the vehicle group. In $\mathbf{E}$, on a well-by-well basis, we quantified the ratio of the average monolayer contraction after vs. before stimulation. We further normalized this ratio to the vehicle group. Each treatment group comprises of measurements performed over $n=7-16$ individual wells of a 96 -well plate. Plotted is the mean and standard error. *indicates $p<0.05$ while ns indicates $p>0.05$ 
[12, 42-46], and supportive dose-response measurements (Supplementary Fig. S1), we picked a sub-maximal dose of $5 \mu \mathrm{M}$ for further evaluation. Individual wells of a single 96well plate (Young's Modulus $=3 \mathrm{kPa}$ ) were pre-treated with either vehicle or Y-27632 for $20 \mathrm{~min}$, and then stimulated further with the barrier disruptive agent, thrombin (1 U/ml, $30 \mathrm{~min})$. Paracellular gaps and monolayer contraction were significantly enhanced by thrombin and diminished by pre-treatment with Y-27632 (Fig. 1B-E). Moreover, these force changes correlated with F-actin cytoskeletal changes, as was revealed by simultaneous measurements of the F-actin cytoskeleton (Supplementary Fig. S2). Specifically, while thrombin enhanced F-actin expression and orientation, these same quantities were significantly reduced by pre-treatment with Y-27632. Finally, the barrier-protective effects of Y-27632 was consistent with ECIS measurements (Supplementary Fig. S3).

Next, we examined biophysical mechanisms of barrier protection for the Tie2 receptor agonist, Angpt-1. In cells, tissue, organs, and animals, Angpt-1 has been demonstrated to counteract permeability and weakening of the endothelial barrier [31]. While roles for actin stress fiber rearrangements, junctional remodeling, and mechanotransduction have been implicated [47-52], in the absence of direct mechanical measurements, the underlying biophysical mechanisms remain unclear. Based on typically used concentrations [35, 48, 53-59], and additional dose-response measurements (Supplementary Fig. S4), we picked a submaximal dose of $300 \mathrm{ng} / \mathrm{ml}$ for further evaluation. Individual wells of a single 96-well plate (Young's Modulus $=3$ $\mathrm{kPa}$ ) were co-stimulated with either vehicle or Angpt-1 together with the barrier disruptive agent, thrombin $(1 \mathrm{U} / \mathrm{ml}$; $30 \mathrm{~min}$ ) (Fig. 2). In accordance with previous findings [35], Angpt-1 co-stimulation reduced thrombin-induced gap formation (Fig. 2A). While this barrier-protective effect, including time-course and efficacy, was recapitulated by ECIS (Fig. 2C, D), it was not correlated with a reduction in cell contractile force (Fig. 2B) or in the orientation and expression of the F-actin cytoskeleton (Supplementary Fig. S2). However, it was associated with an increase in
(A)
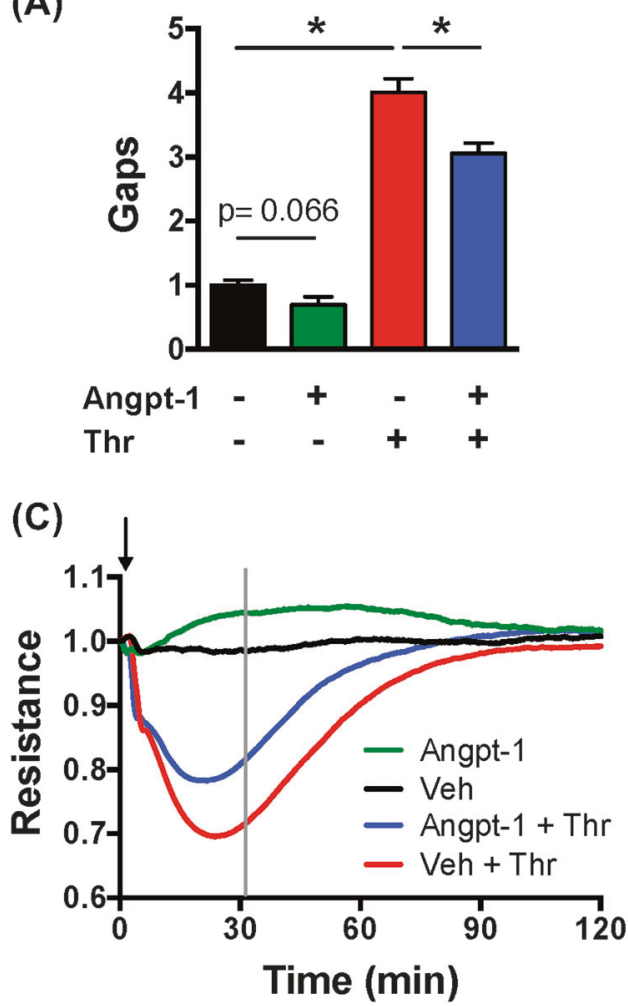

Fig. 2 Angpt-1 reduces thrombin-induced gap formation but does not reduce cellular contractile force. ECs in a 96-well plate were incubated with either vehicle, vehicle + thrombin $(1 \mathrm{U} / \mathrm{ml})$, Angpt-1 $(300 \mathrm{ng} / \mathrm{ml})$, or Angpt-1 + thrombin for $30 \mathrm{~min}$. A While paracellular gaps were reduced by Angpt-1 co-treatment $\mathbf{B}$ cellular contractile forces were not. For gaps, we report changes normalized to vehicle. For contraction, quantities have been normalized to $t=0 \mathrm{~min}$ values and further to the vehicle group. Each treatment group comprises of measurements
(B)

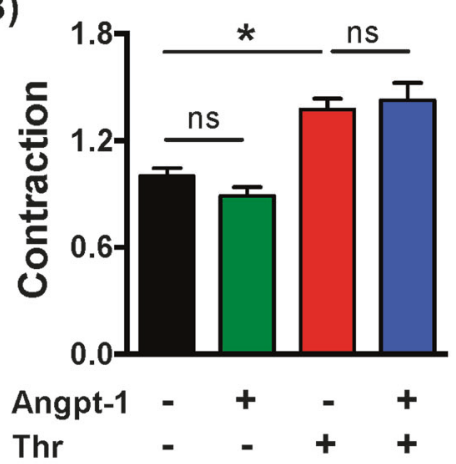

(D)

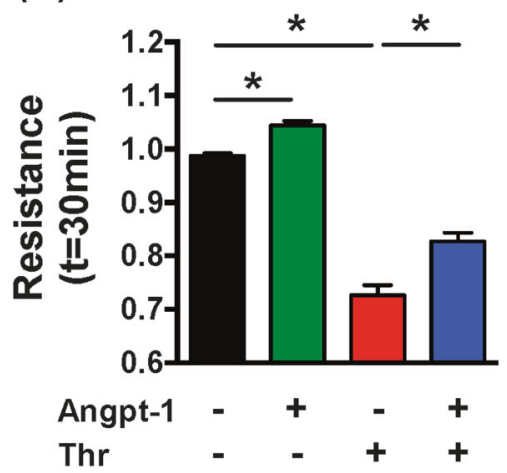

performed over $n=7-16$ individual wells of a 96-well plate. In all bar plots, shown is the mean and standard error. *indicates $p<0.05$. C ECIS measurements confirm mini-XPerT findings. Each curve is averaged over $n=3-5$ individual wells. The gray line marks the time point of $30 \mathrm{~min}$ post treatment; this time point corresponds to gap measurements with mini-XPerT. D Shown are corresponding resistance values. Plotted is the mean and standard error. *indicates $p<0.05$ while ns indicates $p>0.05$ 
VE-cadherin expression at the cell-cell junctions (Supplementary Fig. S5).

To demonstrate the versatility of mini-XPerT, we examined other physiologically-relevant endothelial leak provocateurs that are commonly utilized in barrier function studies: (1) Gram-negative endotoxin lipopolysaccharides (LPS; $100 \mathrm{ng} / \mathrm{ml}, 4 \mathrm{~h}$ ) [48, 60] and (2) the canonical host cytokine, tumor necrosis factor alpha (TNF $\alpha ; 20 \mathrm{ng} / \mathrm{ml}, 4 \mathrm{~h}$ ) $[48,61]$. Notably, their barrier disruptive activity manifests after several hours (e.g., [48, 62]), as opposed to thrombin, whose impact is acute, in the order of minutes (e.g. Fig. 2C). We evaluated these triggers of permeability on soft $(0.3 \mathrm{kPa})$ and stiff $(3 \mathrm{kPa})$ substrates with and without Y-27632 pre-treatment or Angpt-1 co-stimulation (Fig. 3). Paracellular gaps were significantly enhanced by TNF $\alpha /$ LPS stimulation and were largely reduced by the additional presence of Y-27632 or Angpt-1. Correspondingly, Y27632 reduced cell contractile forces (Supplementary Fig. S6) while Angpt-1 did not (Supplementary Fig. S7). Taken together, these findings reveal the generality of the barrierprotective mechanisms of Y-27632 and Angpt-1.

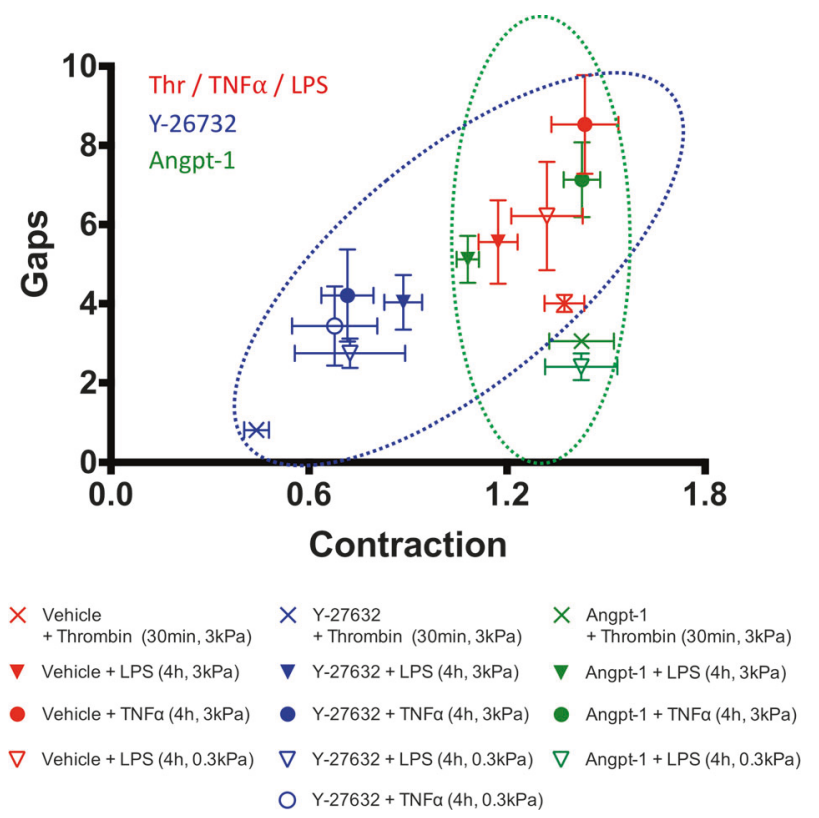

Fig. 3 Barrier protection by Y-27632 is correlated with a reduction of cellular contractile forces, whereas barrier protection by Angpt- 1 is not. Plotted is gaps vs. contraction for the indicated conditions. For gaps, we report changes normalized to vehicle. For contraction, quantities have been normalized to $t=0 \mathrm{~min}$ values and then to the vehicle group. Each treatment group comprises of measurements performed over $n=7-16$ individual wells of a 96-well plate. Plotted is the mean and standard error. Symbols in red, blue, and green correspond to the LPS/TNFo/thrombin, Y-27632 and Angpt-1 treatments, respectively. The ellipses highlight contractile force dependent (blue) or independent (green) reduction of thrombin-, LPS, and TNF $\alpha$ induced gap formation. A lack of concordance for the EC response to $\mathrm{TNF} \alpha$ on the soft substrate remains unclear (Supplementary Fig. S8)
If not through contractile force reduction, how then does Angpt-1 promote barrier defense? This question is of significant interest. Given that Angpt-1 has been shown to counteract endothelial barrier disruption triggered by numerous, unrelated permeability mediators ranging from VEGF to anthrax lethal toxin to thrombin [35, 48], the most widely invoked explanation is that Angpt-1 signaling modulates one or more common downstream effectors of barrier function [31]. We evaluated the two major candidates for this conserved action: (1) F-actin cytoskeletal rearrangement, and (2) VE-cadherin accumulation at the cell-cell junction. We discovered a dominant role for the latter (Supplementary Fig. S5), consistent with the postulated role for Tie2 signaling for VE-cadherin stabilization in vivo [48, 63]. Unexpectedly, Angpt-1 did not prevent agonist-induced enhancement of Factin (Supplementary Fig. S2) or contractile force (Fig. 2 and Supplementary Fig. S2).

In vascular biology, the dogma for EC barrier function has long held that junctional mechanisms are typically coupled to contractile force-dependent F-actin rearrangements. The present results, in contrast, are the first to our knowledge, that uncouple these two cellular processes as they relate to Angpt-1-induced barrier protection. If actin-cytoskeletal rearrangements can be uncoupled from junctional probarrier mechanisms in response to physiological stimuli, this could have implications for other cell types that exert barrier function such as squamous epithelium in the skin, columnar epithelium in the GI tract, and cuboidal epithelium in ducts and acinar structures of secretory organs.

Our data do not rule out the possibility that Angpt-Tie2 signaling is reorganizing forces on a much smaller scale, for example, in the peri-junctional region where the Rho family GTPase Rac1 has been shown to act in ECs [64]. Although beyond the resolution of the current technique, how such localized force reorganization could impact VE-cadherin accumulation at the cell-cell junction remains unclear.

We propose three important future directions for miniXPerT. First, by combining mini-XPerT with monolayer stress microscopy [19], we can also determine intercellular stresses and its consequent effects on gap formation. This will require improved spatial resolution for traction measurements as well as clearly defined monolayer boundaries that can be specified through micropatterning procedures $[12,18,19]$. Second, by imaging gap formation in real-time, we can even more precisely correlate force and gap dynamics. To this end, we are currently evaluating cellular based markers to be able to delineate gaps in real-time. Finally, it would be ideal to combine mini-XPerT with concomitant measurements of molecular signaling.

Acknowledgements This work was supported by the United States National Institute of Health (grant number R56HL133205 to CCH and RK, R21HL123552 and P01HL120839 to RK, R01HL125275 to 
SMP, and R01HL093234 to SMP,R03AII38083 to CCG), the German Research Foundation (DA1209/4-3 to SD), Canadian grants (NSERC RGPIN/05843-2014 to AJE, EQPEQ/472339-2015 to AJE, CIHR Grant \# 143327 to AJE). We thank Prof. Hilmar Stolte and the Biomedical Exchange Program for making the transatlantic cooperation possible. Finally, we thank Yvonne Nicolai and Cleide Da Silva for technical assistance.

\section{Compliance with ethical standards}

Conflict of interest The authors declare that they have no conflict of interest.

\section{References}

1. Sena CM, Pereira AM, Seica R. Endothelial dysfunction - a major mediator of diabetic vascular disease. Biochim Biophys Acta. 2013;1832:2216-31.

2. Ware LB, Matthay MA. The acute respiratory distress syndrome. N Engl J Med. 2000;342:1334-49.

3. Matthay MA, Zimmerman GA, Esmon C, et al. Future research directions in acute lung injury: summary of a National Heart, Lung, and Blood Institute working group. Am J Respir Crit Care Med. 2003;167:1027-35.

4. Patterson CE. Perspectives on lung endothelial barrier function. Adv Mol Cell Biol. 2005;35.

5. Park-Windhol C, D'Amore PA. Disorders of vascular permeability. Annu Rev Pathol. 2016;11:251-81.

6. Zhou C, Townsley MI, Alexeyev M, Voelkel NF, Stevens T. Endothelial hyperpermeability in severe pulmonary arterial hypertension: role of store-operated calcium entry. Am J Physiol Lung Cell Mol Physiol. 2016;311:L560-9.

7. Jamaluddin MS, Wang $\mathrm{X}$, Wang $\mathrm{H}$, et al. Eotaxin increases monolayer permeability of human coronary artery endothelial cells. Arterioscler Thromb Vasc Biol. 2009;29:2146-52.

8. Phinikaridou A, Andia ME, Lavin B, et al. Increased vascular permeability measured with an albumin-binding magnetic resonance contrast agent is a surrogate marker of rupture-prone atherosclerotic plaque. Circ Cardiovasc Imaging. 2016;9:e004910.

9. Majno G, Palade GE. Studies on inflammation. 1. The effect of histamine and serotonin on vascular permeability: an electron microscopic study. J Biophys Biochem Cytol. 1961;11:571-605.

10. Majno G, Palade GE, Schoefl GI. Studies on inflammation. II. The site of action of histamine and serotonin along the vascular tree: a topographic study. J Biophys Biochem Cytol. 1961;11:607-26.

11. Majno G, Shea SM, Leventhal M. Endothelial contraction induced by histamine-type mediators: an electron microscopic study. J Cell Biol. 1969;42:647-72.

12. Krishnan R, Klumpers DD, Park CY, et al. Substrate stiffening promotes endothelial monolayer disruption through enhanced physical forces. Am J Physiol Cell Physiol. 2011;300:C146-54.

13. Beckers CM, Knezevic N, Valent ET, et al. ROCK2 primes the endothelium for vascular hyperpermeability responses by raising baseline junctional tension. Vasc Pharmacol. 2015;70:45-54.

14. Valent ET, van Nieuw Amerongen GP, van Hinsbergh VW, Hordijk PL. Traction force dynamics predict gap formation in activated endothelium. Exp Cell Res. 2016;347:161-70.

15. Dudek SM, Garcia JG. Cytoskeletal regulation of pulmonary vascular permeability. J Appl Physiol. 2001;91:1487-500.

16. Birukova AA, Arce FT, Moldobaeva N, et al. Endothelial permeability is controlled by spatially defined cytoskeletal mechanics: atomic force microscopy force mapping of pulmonary endothelial monolayer. Nanomedicine. 2009;5:30-41.
17. Stevens T, Garcia JG, Shasby DM, Bhattacharya J, Malik AB. Mechanisms regulating endothelial cell barrier function. Am J Physiol Lung Cell Mol Physiol. 2000;279:L419-22.

18. Hardin CC, Rajendran K, Manomohan G, et al. Glassy dynamics, cell mechanics and endothelial permeability. J Phys Chem B. 2013;117:12850-6.

19. Hardin CC, Chattoraj J, Manomohan G, et al. Long-range stress transmission guides endothelial gap formation. Biochem Biophys Res Commun. 2017;495:749-54.

20. Liu Z, Tan JL, Cohen DM, et al. Mechanical tugging force regulates the size of cell-cell junctions. Proc Natl Acad Sci USA. 2010;107:9944-9.

21. Lampi MC, Faber CJ, Huynh J, et al. Simvastatin ameliorates matrix stiffness-mediated endothelial monolayer disruption. PLoS ONE. 2016;11:e0147033.

22. Urbano RL, Furia C, Basehore S, Clyne AM. Stiff substrates increase inflammation-induced endothelial monolayer tension and permeability. Biophys J. 2017;113:645-55.

23. Maruthamuthu V, Sabass B, Schwarz US, Gardel ML. Cell-ECM traction force modulates endogenous tension at cell-cell contacts. Proc Natl Acad Sci USA. 2011;108:4708-13.

24. Tzima E, Irani-Tehrani M, Kiosses WB, et al. A mechanosensory complex that mediates the endothelial cell response to fluid shear stress. Nature. 2005;437:426-31.

25. Bischoff I, Hornburger MC, Mayer BA, et al. Pitfalls in assessing microvascular endothelial barrier function: impedance-based devices versus the classic macromolecular tracer assay. Sci Rep. 2016;6:23671.

26. Trepat $X$, Wasserman MR, Angelini TE, et al. Physical forces during collective cell migration. Nat Phys. 2009;5:426-30.

27. Dubrovskyi O, Birukova AA, Birukov KG. Measurement of local permeability at subcellular level in cell models of agonist- and ventilator-induced lung injury. Lab Invest. 2012;93:254-63.

28. Park CY, Zhou EH, Tambe D, et al. High-throughput screening for modulators of cellular contractile force. Integr Biol (Camb). 2015;7:1318-24

29. Yoshie H, Koushki N, Kaviani R, et al. Traction force screening enabled by compliant PDMS elastomers. Biophys J. 2018;114:2194-9.

30. van Nieuw Amerongen GP, Musters RJ, Eringa EC, Sipkema P, van Hinsbergh VW. Thrombin-induced endothelial barrier disruption in intact microvessels: role of RhoA/Rho kinase-myosin phosphatase axis. Am J Physiol Cell Physiol. 2008;294:C1234-41.

31. Milam KE, Parikh SM. The angiopoietin-Tie2 signaling axis in the vascular leakage of systemic inflammation. Tissue Barriers. 2015;3:e957508.

32. Krishnan R, Tambe D, Steward RL, Hardin C, Butler J. Traction microscopy cells, forces, and the microenvironment. ch 4, pp. 75-96 2015;ISBN (Hardcover): 978-981-4613-36-1.

33. Butler JP, Tolic-Norrelykke IM, Fabry B, Fredberg JJ. Traction fields, moments, and strain energy that cells exert on their surroundings. Am J Physiol Cell Physiol. 2002;282:C595-605.

34. Boudaoud A, Burian A, Borowska-Wykret D, et al. FibrilTool, an ImageJ plug-in to quantify fibrillar structures in raw microscopy images. Nat Protoc. 2014;9:457-63.

35. David S, Ghosh CC, Mukherjee A, Parikh SM. Angiopoietin-1 requires IQ domain GTPase-activating protein 1 to activate Rac1 and promote endothelial barrier defense. Arterioscler Thromb Vasc Biol. 2011;31:2643-52.

36. van Nieuw Amerongen GP, Beckers CM, Achekar ID, et al. Involvement of Rho kinase in endothelial barrier maintenance. Arterioscler Thromb Vasc Biol. 2007;27:2332-9.

37. van Nieuw Amerongen GP, Vermeer MA, Negre-Aminou P, et al. Simvastatin improves disturbed endothelial barrier function. Circulation. 2000;102:2803-9. 
38. Essler M, Amano M, Kruse HJ, et al. Thrombin inactivates myosin light chain phosphatase via Rho and its target Rho kinase in human endothelial cells. J Biol Chem. 1998;273:21867-74.

39. Amano M, Chihara K, Kimura K, et al. Formation of actin stress fibers and focal adhesions enhanced by Rho-kinase. Science. 1997;275:1308-11.

40. Kimura K, Ito M, Amano M, et al. Regulation of myosin phosphatase by Rho and Rho-associated kinase (Rho-kinase) [see comments]. Science. 1996;273:245-8.

41. Riento K, Guasch RM, Garg R, Jin B, Ridley AJ. RhoE binds to ROCK I and inhibits downstream signaling. Mol Cell Biol. 2003;23:4219-29.

42. Carbajal JM, Gratrix ML, Yu CH, Schaeffer RC Jr. ROCK mediates thrombin's endothelial barrier dysfunction. Am J Physiol Cell Physiol. 2000;279:C195-204.

43. Okumura N, Inoue R, Okazaki Y, et al. Effect of the Rho kinase inhibitor Y-27632 on corneal endothelial wound healing. Invest Ophthalmol Vis Sci. 2015;56:6067-74.

44. Wojciak-Stothard B, Potempa S, Eichholtz T, Ridley AJ. Rho and Rac but not $\mathrm{Cdc} 42$ regulate endothelial cell permeability. J Cell Sci. 2001;114:1343-55.

45. Fleming I, Fisslthaler B, Busse R. Calcium signaling in endothelial cells involves activation of tyrosine kinases and leads to activation of mitogen-activated protein kinases. Circ Res. 1995;76:522-9.

46. Peh GS, Adnan K, George BL, et al. The effects of Rho-associated kinase inhibitor Y-27632 on primary human corneal endothelial cells propagated using a dual media approach. Sci Rep. 2015;5:9167.

47. Hakanpaa L, Sipila T, Leppanen VM, et al. Endothelial destabilization by angiopoietin-2 via integrin betal activation. Nat Commun. 2015;6:5962.

48. Ghosh CC, David S, Zhang R, et al. Gene control of tyrosine kinase TIE2 and vascular manifestations of infections. Proc Natl Acad Sci USA. 2016;113:2472-7.

49. Cascone I, Audero E, Giraudo E, et al. Tie-2-dependent activation of RhoA and Rac1 participates in endothelial cell motility triggered by angiopoietin-1. Blood. 2003;102:2482-90.

50. Mammoto T, Parikh SM, Mammoto A, et al. Angiopoietin-1 requires p190 RhoGAP to protect against vascular leakage in vivo. J Biol Chem. 2007;282:23910-8.

51. Fukuhara S, Sako K, Minami T, et al. Differential function of Tie2 at cell-cell contacts and cell-substratum contacts regulated by angiopoietin-1. Nat Cell Biol. 2008;10:513-26.
52. Saharinen P, Eklund L, Miettinen J, et al. Angiopoietins assemble distinct Tie2 signalling complexes in endothelial cell-cell and cellmatrix contacts. Nat Cell Biol. 2008;10:527-37.

53. Li X, Stankovic M, Bonder CS, et al. Basal and angiopoietin-1mediated endothelial permeability is regulated by sphingosine kinase-1. Blood. 2008;111:3489-97.

54. Seegar TC, Eller B, Tzvetkova-Robev D, et al. Tie1-Tie2 interactions mediate functional differences between angiopoietin ligands. Mol Cell. 2010;37:643-55.

55. Abdel-Malak NA, Harfouche R, Hussain SN. Transcriptome of angiopoietin 1-activated human umbilical vein endothelial cells. Endothelium. 2007;14:285-302.

56. Harfouche R, Gratton JP, Yancopoulos GD, et al. Angiopoietin-1 activates both anti- and proapoptotic mitogen-activated protein kinases. FASEB J. 2003;17:1523-5.

57. Echavarria R, Mayaki D, Neel JC, et al. Angiopoietin-1 inhibits toll-like receptor 4 signalling in cultured endothelial cells: role of miR-146b-5p. Cardiovasc Res. 2015;106:465-77.

58. Echavarria R, Hussain SN. Regulation of angiopoietin-1/Tie-2 receptor signaling in endothelial cells by dual-specificity phosphatases 1, 4, and 5. J Am Heart Assoc. 2013;2:e000571.

59. Ghosh CC, Mukherjee A, David S, et al. Angiopoietin-1 requires oxidant signaling through $\mathrm{p} 47$ phox to promote endothelial barrier defense. PLoS One. 2015;10:e0119577.

60. Goldblum SE, Ding X, Brann TW, Campbell-Washington J. Bacterial lipopolysaccharide induces actin reorganization, intercellular gap formation, and endothelial barrier dysfunction in pulmonary vascular endothelial cells: concurrent F-actin depolymerization and new actin synthesis. $\mathrm{J}$ Cell Physiol. 1993;157:13-23.

61. Goldblum SE, Ding X, Campbell-Washington J. TNF-alpha induces endothelial cell F-actin depolymerization, new actin synthesis, and barrier dysfunction. Am J Physiol. 1993;264: C894-905.

62. Gong H, Gao X, Feng S, et al. Evidence of a common mechanism of disassembly of adherens junctions through Galpha13 targeting of VE-cadherin. J Exp Med. 2014;211:579-91.

63. Frye M, Dierkes M, Kuppers V, et al. Interfering with VE-PTP stabilizes endothelial junctions in vivo via Tie-2 in the absence of VE-cadherin. J Exp Med. 2015;212:2267-87.

64. Timmerman I, Heemskerk N, Kroon J, et al. A local VE-cadherin and Trio-based signaling complex stabilizes endothelial junctions through Rac1. J Cell Sci. 2015;128:3041-54. 